\title{
WHICH CARBON DERIVATIVES ARE APPLICABLE IN PRACTICE? A CASE STUDY OF A EUROPEAN STEEL COMPANY
}

\author{
MARTin ŠmíD, FrantišEK Zapletal AND JANA HANČLOvÁ
}

This paper constructs and analyses a model for optimal production and emission covering of a real-life European steel company. The emissions may be covered by a combination of EUA and CER allowances and their derivatives. The company is assumed to be risk-averse, maximizing the Mean-CVaR criterion. The problem is analysed given continuum of risk-aversion coefficients and three scenarios of the demand.

It is found that the production does not depend on the risk aversion and is always maximal, but the optimal composition of the (spot) allowances and their derivatives depends non-trivially on both the risk aversion and the demand. Out of all the derivatives, only futures are used. Surprisingly, options are never used.

Keywords: carbon allowances, carbon derivatives, mean-CVaR, optimization

Classification: $90 \mathrm{~B} 30,91 \mathrm{~B} 28$

\section{INTRODUCTION}

For more than ten years, European companies emitting carbon dioxide $\left(\mathrm{CO}_{2}\right)$ are obliged to cover their emissions by emission allowances [5], namely by the European Union Allowances (EUAs), which can be, up to certain limit, substituted by the Certified Emission Reductions (CERs) 3, 5. Certain amounts of the EUAs are granted to the companies for free, additional allowances have to be bought, either in auctions or on secondary markets.

Numerous derivatives of both the EUAs and CERs are available on secondary markets. Our goal is to answer the question whether and how the derivatives are helpful for real-life companies.

Many papers on the carbon allowances, their derivatives, and relationships between them have been published $([2,6,19,11,12])$. Less attention has been paid to the applicability of the derivatives from the point of view of individual companies. Some works, such as 7], study the situation of particular companies and count with the derivatives, namely the futures. No study yet, however, tried to determine which combinations of the spots (the allowances themselves) and various derivatives are optimal.

Without any deep analysis, it is clear that the derivatives may be used to reduce risk. Call options, for instance, can help companies to hedge against the risk of high spot 
prices. Further, as the allowances have to be submitted as late as at the end of each year, it could be advantageous to buy futures rather than risk buying the spots at the end of the year, or block the capital by buying the spots at the beginning of the year. It is, however, unclear, what proportion of the derivatives, possibly together with the spots, reduces the risk the most.

To help to answer this question, we model the situation of a real-life Czech steel company, which has to decide on its production and simultaneously on the way of covering their emissions with respect to the Mean-CVaR decision criterion 11 To cover the emissions, the company may use EUA and CER spots, EUA and CER futures, and various EUA options ${ }^{2}$

We solve the company's decision problem for various risk aversion parameters and for three possible levels of the demand for the production of the company. As a result, we find that EUA futures are always used to secure the EUAs. Meanwhile, the maximum number of the (cheaper) CERs is always bought. In addition, the model recommends to speculate within the bounds we gave it, given that the risk aversion level is low. Interestingly, no options and no CER futures are used under any circumstances.

Our paper is organized as follows. After this introduction, Section 2 gives the definition of the decision model. In Section 3, we describe the data we use to construct the stochastic model for the spot and future prices. In Section 4, we discuss the computation of the decision model's solution. In Section 5, the results of the analysis are presented. After that, the results are discussed and the paper is concluded. The procedure of the linearization of the decision problem is described in the Appendix.

\section{DECISION MODEL}

\subsection{Assumptions}

In the present subsection, we formulate assumptions underlying our model, which we group into categories.

\section{Production}

There is only one (unit) decision period.

There are $n$ products produced, some of them serving as inputs for further production.

The demand for the products is given by $\boldsymbol{d} \in \mathbb{R}_{+}^{n}$, their selling prices are determined by $\boldsymbol{p} \in \mathbb{R}_{+}^{n}$ and the unit production costs (of the final products) are given by $\boldsymbol{c} \in \mathbb{R}_{+}^{n}$. All $\boldsymbol{d}, \boldsymbol{p}$ and $\boldsymbol{c}$ all known to the decision maker at the beginning of the period.

The amount $\boldsymbol{x} \in \mathbb{R}_{+}^{n}$ of the inputs which are necessary for final production $\boldsymbol{y} \geq 0$ is given by $\boldsymbol{x}=\boldsymbol{T} \boldsymbol{y}$ where $\boldsymbol{T} \in \mathbb{R}^{n \times n}$ is an inverted technological matrix (i. e. $\boldsymbol{T}^{-1}$ is the matrix, given by the technological process, transforming inputs into outputs).

\footnotetext{
${ }^{1}$ We chose CVaR as a risk part of the criterion because it is one-sided, coherent and easily integrable into the optimization problem (see [1], 10], respectively).

${ }^{2}$ We did not include CER options because no options with the desired maturity were available on the market we took data from.
} 
The production limits are given by vector $\boldsymbol{w} \geq 0$, i. e.,

$$
\boldsymbol{y} \geq \mathbf{0}, \quad \boldsymbol{T} \boldsymbol{y} \leq \boldsymbol{w} .
$$

\section{Emissions}

The amount of $\mathrm{CO}_{2}$ resulting from the production of inputs $\boldsymbol{x}$ is given by $\boldsymbol{h}^{T} \boldsymbol{x}$ where $\boldsymbol{h} \in \mathbb{R}^{n}$ is a vector of emissions per ton of individual products.

The vector of the emissions of the non-carbon greenhouse gases $\left(\mathrm{SO}_{2}, \mathrm{NO}_{x}\right.$ and the air-born dust) from production $\boldsymbol{x}$ is given by $\boldsymbol{H} \boldsymbol{x}$ where $\boldsymbol{H} \in \mathbb{R}^{3 \times n}$ is a matrix, analogous to $\boldsymbol{h}$.

The constraints of the emissions, given by legislation, are given by

$$
\boldsymbol{H T y} \leq \varsigma, \quad(\boldsymbol{H}, \boldsymbol{h}) \boldsymbol{T} \boldsymbol{y} \leq \boldsymbol{\mu} .
$$

Here, $\boldsymbol{\varsigma}$ are "caps" and $\boldsymbol{\mu}$ are limits ${ }^{3}$

\section{Finance}

The revenues from the final production are collected at the end of the period, i. e. at $t=1$.

The production costs are funded at $t=0$ by a credit with a low interest rate $\iota$.

The remaining costs, namely those of financial operations, are funded by loans payable at $t=1$ with an interest rate $\rho>\iota$.

If there is some excess cash at $t=0$, then it may be deposited up to $t=1$ with the interest rate $\iota$.

The insufficiency of the unit of cash at $t=1$ is penalized by a constant $\sigma$, which may be thus understood as a prohibitive interest rate.

\section{Allowances}

$r$ EUA allowances are obtained for free.

At $t=0$, the company possibly buys

$-s_{0}^{E}$ EUA spots, i. e. the permits themselves,

$-f^{E}$ EUA futures 4

$-\phi_{1}, \phi_{2}, \ldots \phi_{k}$ call EUA options 5 with strike prices $K_{1}<K_{2}<\cdots<K_{k}$, respectively,

\footnotetext{
3 The "caps" are yearly limits setting maximum amounts of selected gas emissions, the limits are maximum amounts for half an hour, see [13] for details.

${ }^{4}$ Future with maturity $T$ is the commitment to buy the spot at $T$ for a fixed price (future price).

${ }^{5}$ Call/put option with maturity $T$ is the right (not obligation) to buy/sell the spot for a given price (strike price) at $T$.
} 
- $\psi_{1}, \psi_{2}, \ldots \psi_{l}$ put EUA options with strike prices $L_{1}>L_{2}>\cdots>L_{l}$, respectively,

$-s_{0}^{C}$ CER spots,

$-f^{C}$ CER futures.

Short sales are not allowed:

$$
s_{0}^{E} \geq-r, f^{E} \geq 0, \phi \geq 0, \psi \geq 0, s_{0}^{C} \geq 0, f^{C} \geq 0 .
$$

At $t=1$, the company possibly buys $s_{1}^{E}$ EUA spots and $s_{1}^{C}$ CER spots, short sales are not allowed:

$$
s_{0}^{C}+s_{1}^{C} \geq 0, \quad r+s_{0}^{E}+s_{1}^{E} \geq 0 .
$$

Banking of the permits for subsequent periods is not allowed, i. e. the total number of permits has to be equal to the total amount of the carbon emissions:

$$
r+s_{0}^{E}+s_{0}^{C}+s_{1}^{E}+s_{1}^{C}+f^{E}+f^{C}+\sum_{i=1}^{k} \phi_{i}-\sum_{i=1}^{l} \psi_{i}=\boldsymbol{h} \boldsymbol{T} \boldsymbol{y} .
$$

Only a limited number of CERs may be applied according to the legislation, in particular,

$$
s_{0}^{C}+s_{1}^{C}+f^{C} \leq \eta \boldsymbol{h} \boldsymbol{T} \boldsymbol{y}
$$

where $\eta=0.16$

In accordance with the usual practice, margin $\zeta_{E}$ is required when holding an EUA future. In particular, the difference of the current and the initial price of the future plus a margin $\zeta$ has to always be deposited at the exchange where the futures are traded. Consequently, the costs of an EUA future maintenance amount to

$$
M^{E}=\sum_{j=0}^{\delta} \rho_{\delta}\left[p_{0}^{E, f}-\left(P_{j / d}^{E, f}+\eta_{E}\right)\right]^{+}
$$

where $\delta$ is the number of business days within the time period, $\rho_{\delta}=(1+\rho)^{1 / \delta}-1$ is the $1 / \delta$ time units interest rate, $p_{0}^{E, f}$ is the EUA future price at $t=0$, and $P_{\tau}^{E, f}$ is the (random) EUA future price at $\tau$. For more details on the mechanism of future markets, see [8].

Margin $\zeta_{C}$ is required when holding a CER future, and the costs $M^{C}$ of the CER future maintenance are calculated analogously to the EUA ones.

The company does not speculate: in particular, they would neither buy more permits than needed at $t=0$ nor they would not buy more put options than the initial number of spots, i. e.,

$$
s_{0}^{E}+s_{0}^{C}+f^{E}+f^{C}+\sum_{i=1}^{k} \phi_{1} \leq \boldsymbol{h} \boldsymbol{T} \boldsymbol{y}, \quad s_{0}^{C} \leq \eta \boldsymbol{h} \boldsymbol{T} \boldsymbol{y}, \quad \sum \psi_{i} \leq r .
$$

\footnotetext{
${ }^{6}$ EU Directive 2003/87/EC
} 


\subsection{Model Definition}

As it follows from the assumptions, the vector of the decision variables is $(\boldsymbol{y}, \boldsymbol{\xi})$ where

$$
\boldsymbol{\xi}=\left(s_{0}^{E}, s_{0}^{C}, s_{1}^{E}, s_{1}^{C}, f^{E}, f^{C}, \phi_{1}, \ldots, \phi_{k}, \psi_{1}, \ldots, \psi_{l}\right)
$$

The individual parts of the company's cash flow are following:

The gross balance of emissions trading at $t=0$, excluding margins of futures, equals to

$$
E_{0}(\boldsymbol{\xi})=-s_{0}^{E} p_{0}^{E}-s_{0}^{C} p_{0}^{C}-\sum_{i=1}^{k} \phi_{i} p_{0}^{c, K_{i}}-\sum_{i=1}^{l} \psi_{i} p_{0}^{p, L_{i}}
$$

where $p_{0}^{E}$ and $p_{0}^{C}$ are EUA, CER, respectively, prices at $t=0, p_{0}^{c, K}$ is the price of the call EUA option with strike price $K$ at $t=0$ and $p_{0}^{p, L}$ is the price of the put EUA option with strike price $L$ at $t=0$.

The costs of the futures maintenance comes out as

$$
F(\boldsymbol{\xi})=F\left(\boldsymbol{\xi} ; M^{E}, M^{C}\right)=f^{E} M^{E}+f^{C} M^{C} .
$$

The cash balance at $t=1$ resulting from the production is

$$
B(\boldsymbol{y})=\boldsymbol{p}^{T} \min (\boldsymbol{d}, \boldsymbol{y})-(1+\iota) \boldsymbol{c}^{T} \boldsymbol{y}
$$

The balance resulting from the emissions trading at $t=1$ is

$$
\begin{aligned}
& E_{1}(\boldsymbol{\xi})=E_{1}\left(\boldsymbol{\xi} ; P_{1}^{E}, P_{1}^{C}\right) \\
= & -s_{1}^{E} P_{1}^{E}-s_{1}^{C} P_{1}^{C}-f^{E} p_{0}^{E, f}-f^{C} p_{0}^{C, f}-\sum_{i=1}^{k} \phi_{i} \min \left(P_{1}^{E}, K_{i}\right)+\sum_{i=1}^{l} \psi_{i} \max \left(P_{1}^{E}, L_{i}\right) .
\end{aligned}
$$

Taking the interests and penalizations into account, the value function of our decision problem is given by

$$
V(\boldsymbol{y}, \boldsymbol{\xi})=V\left(\boldsymbol{y}, \boldsymbol{\xi} ; P_{1}^{E}, P_{1}^{C}, M^{E}, M^{C}\right)=g_{1+\sigma, 1}\left(g_{1+\rho, 1+\iota}\left(E_{0}\right)-F+E_{1}+B\right)
$$

where

$$
g_{\alpha, \beta}(x)= \begin{cases}\beta x & x \geq 0 \\ \alpha x & x \leq 0\end{cases}
$$

for any non-negative $\alpha$ and $\beta$.

The decision problem itself is then formulated as

$$
\begin{array}{ll}
\max _{\boldsymbol{y}, \boldsymbol{\xi}} & (1-\lambda) \mathbb{E} V(\boldsymbol{y}, \boldsymbol{\xi})-\lambda \mathrm{CVaR}_{5 \%}(-V(\boldsymbol{y}, \boldsymbol{\xi})) \\
& \text { s.t. }(P),(L),(F),(G),(E),(C),(S)
\end{array}
$$

where $0 \leq \lambda \leq 1$ is a level of risk aversion. 


\subsection{Parameters}

The parameters $\boldsymbol{c}, \boldsymbol{p}, \boldsymbol{d}, \boldsymbol{T}, \boldsymbol{w}, \boldsymbol{H}$ and $\boldsymbol{h}$, which are related to the production and sales, were taken from a real-life Czech steel company. The values of the parameters are the same as those in [13] except for $\boldsymbol{d}$, which is deterministic in the present paper (see Section 4). According to our agreement with the company, we do not publish these values. The number of EUAs granted for free is $r=145,098$. The interest rates $\iota, \rho$ and $\sigma$ were set to $0.015,0.04$ and 0.15 , respectively, according to an anonymous expert economist.

The time of the decision $(t=0)$ was set to February 29th, 2016, and the end of the period $(t=1)$ was set to February 28 th, 2017, i. e. one day before the allowances have to be delivered.

\section{DATA}

\subsection{Description}

The prices (in EUR) of the allowances and their derivatives were taken from the website of ICE, which is one of the secondary markets where the allowances are traded (http: //www.theice.com). The daily series of the spot prices and the prices of their futures with maturity on March 1st, 2017 are depicted in Figures 1 and 2

It is clearly seen from the graphs that, while the EUA prices behave in a "standard financial" way, the price series of the CER spots is inhomogeneous, exhibiting a clear change point on March 31st, 2015, which was the last day when the CERs from the first commitment period of the Kyoto protocol could be exchanged for EUAs (see [4]). At that time, the price jumped up dramatically and, subsequently, the series calmed down. Interestingly, the futures did not exhibit any analogous change, which is probably because the change was anticipated by the markets.

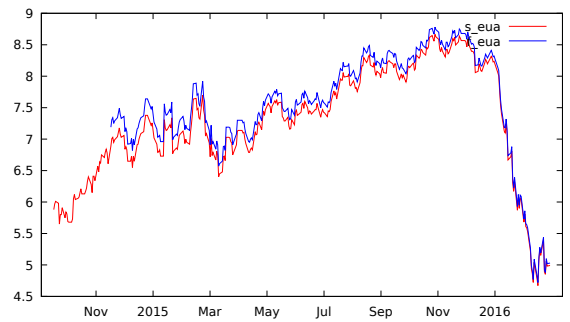

(a) Spots and futures.

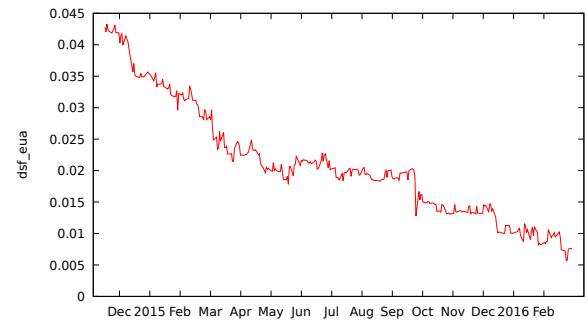

(b) Difference of spots and futures.

Fig. 1. EUA spots and futures with maturity March 2017.

The descriptive statistics of the log-returns of all four series may be found in Table 1 . For the CER spots, the statistics were computed only from the post-change period.

The prices of the spots, futures, and selected EUA options ${ }^{8}$ from February 29th, 2016 $(t=0)$ are listed in Table 2 ,

\footnotetext{
${ }^{8}$ The maturity of the options is March 1st, 2017.
} 


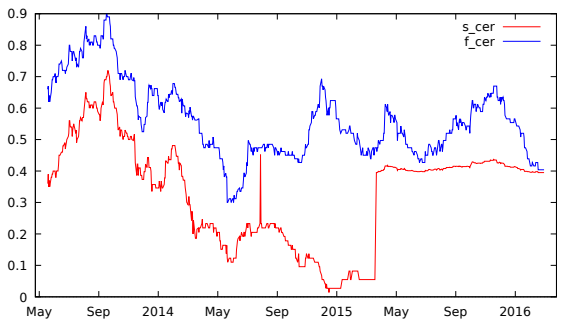

(a) Spots and Futures

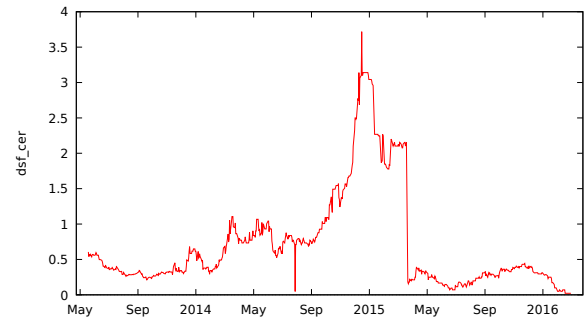

(b) Difference of Spots and Futures

Fig. 2. CER spots and futures with maturity March 2017.

\begin{tabular}{l|clll} 
Variable & Mean & Volatility & Minimum & Maximum \\
\hline$\Delta \log \left(P^{E}\right)$ & 0.00049 & 0.43 & -0.17 & 0.023 \\
$\Delta \log \left(F^{E}\right)$ & -0.0027 & 0.33 & -0.10 & 0.083 \\
$\Delta \log \left(P^{C}\right)$ & -0.000043 & 0.071 & -0.017 & 0.71 \\
$\Delta \log \left(F^{C}\right)$ & -0.00070 & 0.45 & -0.24 & 0.10
\end{tabular}

Tab. 1. Descriptive statistics.

\begin{tabular}{l|l|l|l}
\multicolumn{2}{l|}{ Spots } & \multicolumn{2}{l}{ Futures } \\
\hline$p_{0}^{E}$ & 4.992 & $f_{0}^{E}$ & 5.030 \\
$p_{0}^{C}$ & 0.395 & $f_{0}^{C}$ & 0.404
\end{tabular}

\begin{tabular}{l|lllll} 
EUA options \\
Strike price & 3.00 & 4.00 & 5.00 & 6.00 & 7.00 \\
\hline Calls $\left(p_{0}^{c}\right)$ & 2.31 & 1.95 & 1.12 & 0.77 & 0.53 \\
Puts $\left(p_{0}^{p}\right)$ & 0.15 & 0.56 & 1.06 & 1.71 & 2.48
\end{tabular}

Tab. 2. Prices at $t=0$.

\subsection{Stochastic Model}

As the primary purpose of the present paper was not to conduct any detailed econometric analysis of the prices, we used only a simple stochastic model for the spots and their derivatives. In particular, we supposed the daily log returns of the spots to follow the $\mathrm{ARCH}(1)$ processes:

$$
\Delta \log \left(P_{t}\right)=\sigma_{t} \epsilon_{t}, \quad \sigma_{t}^{2}=\alpha_{0}+\alpha_{1}\left(\Delta \log \left(P_{t-1}\right)\right)^{2},
$$

where $\epsilon_{t}$ are independent standard Gaussian ${ }^{9}$ Further, being inspired by Figure 1 (b), which suggests the convergence of the future and spot prices on the maturity date, we chose to describe the futures prices by a cost-of-carry model with a log $\mathrm{AR}(1)$ noise:

$$
F_{t}=P_{t} \exp \left\{\gamma(t-\tau) \eta_{t}\right\}, \quad \log \left(\eta_{t}\right)=\beta_{0}+\beta_{1} \log \left(\eta_{t-1}\right)+\sigma \nu_{t}
$$

\footnotetext{
${ }^{9}$ Unlike the rest of the paper, the time unit is the business day in the present Section.
} 
where $\nu_{t}$ are independent standard Gaussian and $\tau$ is the maturity time. The noise processes of the individual price series were assumed to be mutually independent.

The parameters of (3) were estimated by the standard $\mathrm{ARCH}$ estimation techniques, and the parameters of (4) were estimated by OLS applied to the equation

$$
\begin{array}{r}
\log \left(D_{t}\right)=\phi+\beta_{0} \log \left(D_{t-1}\right)+\sigma \nu_{t} \\
D_{t}=\log F_{t}-\log P_{t}, \quad \phi=\beta_{0}+\left(1-\beta_{1}\right) \log (\gamma),
\end{array}
$$

which stems from a transformation of (4). The fact that parameters $\gamma$ and $\beta_{0}$ are not identified does not play any role here because our knowledge of $\phi$ suffices for the Monte Carlo simulation of the margin values. The results of the estimation, in which all parameters came out significant, are listed in Table 3.

\begin{tabular}{c|ccccc} 
& $\alpha_{0}$ & $\alpha_{1}$ & $\phi$ & $\beta_{1}$ & $\sigma$ \\
\hline EUA & $0.00055(0.00003)$ & $0.23(0.06)$ & $-0.17(0.07)$ & $0.96(0.02)$ & 0.22 \\
CER & $0.00002(0.000002)$ & $0.31(0.12)$ & $0.04(0.02)$ & $0.98(0.01)$ & 0.87
\end{tabular}

Tab. 3. Parameters of the stochastic model.

The futures margins were, according to the rules of the market, set to the $99 \%$ quantiles of the two-day price changes, computed under the assumption of geometrically Brownian prices, with the resulting values $\zeta_{E}=0.067, \zeta_{C}=0.103$.

\section{PROBLEM SOLUTION}

As we did not want our results to be dependent on the level of the risk aversion and/or on the particular situation on the steel products market, we solved (2) gradually for a sufficient number of values of $\lambda$, ranging from 0 to 1 , and for three different values of the demand $d$ determined similarly as in 13,10

As the expectation in problem (2) is intractable, we approximated it using a Monte Carlo estimate based on a random sample of 1023 realizations of random vector $\left(P_{1}^{E}, M^{E}\right.$, $P_{1}^{C}, M^{C}$ ). The characteristics of the sample are listed in Table 4.

\begin{tabular}{l|ccc} 
Variable & Mean & Std. dev. & Volatility \\
\hline$P_{1}^{E}$ & 5.497 & 2.650 & 0.531 \\
$P_{1}^{C}$ & 0.396 & 0.029 & 0.073 \\
$M^{E}$ & 0.026 & 0.024 & \\
$M^{C}$ & 0.001 & 0.001 &
\end{tabular}

Tab. 4. Monte Carlo sample.

\footnotetext{
${ }^{10}$ In particular, the middle scenario of the demand was set to the current sales of the company, the low one was taken equal to the lowest scenario from 13 while the highest was set to the highest one from there.
} 
As, according to Appendix A, the Monte Carlo approximation of problem (2) may be linearised, we could solve the individual instances of (2) by a linear programming solver. To automate the process of repeated solving, we run a GAMS script invoking the solver for each $\lambda$ and each demand scenario.

\section{RESULTS}

As neither the production limits nor the emission limits were reached under any of the demand scenarios and as the profit from the production including the emission costs is positive, it was optimal to produce everything what was demanded under any combination of the risk aversion and the demand. The amounts of the allowances needed given the individual demand scenarios are listed in Table 5.

\begin{tabular}{l|ccc} 
Scenario & Low & Middle & High \\
\hline Total needed & 121,309 & 191,649 & 256,548 \\
Granted for free & & 145,098 & \\
Additional needed & $-23,789$ & 46,551 & 111,450
\end{tabular}

Tab. 5. Amounts of allowances needed.

As for the optimal values of the "financial" variables $\xi$, some combination of the spots and the futures came out as optimal in all the cases. The options, on the other hand, were never used.

The optimal compositions are depicted in Figure 3 and Figure 4.

Given all the risk aversion levels and all the scenarios, the maximum allowed number of the (cheap) CERs was always bought, either at the beginning (higher $\lambda s$ ) or at the end (lower $\lambda \mathrm{s}$ ); to see it, note that the curves in the CER spot graphs add up to constants. Surprisingly, no CER futures were bought even in cases when the spots were bought at $t=111$

For $\lambda \leq 0.27$, the model recommends to speculate with the EUAs. In particular, it recommends to sell the spots obtained for free and buy the futures to exploit the rise of the EUAs expected price. The spots are sold at the end of the period (given $\lambda \leq 0.13)$ or at the beginning $(0.13<\lambda<0.23)$. Some EUA spots are also bought at the beginning $(\lambda<0.1)$. Interestingly, the strategy of the speculation coincides for all the three scenarios.

For $\lambda \geq 0.23$, no speculation appears and the futures are always used to buy the EUAs if they are missing. Under the low demand scenario, the redundant allowances are sold at the beginning.

The detailed results, as well as the Gretl script for the stochastic model estimation and the LibreOffice spreadsheet computing some intermediary results and the scenarios may be found at https://github.com/utia-econometrics/szh2016.

\footnotetext{
${ }^{11}$ Interestingly, the CER futures started to be used after we banned the EUA futures from the model.
} 


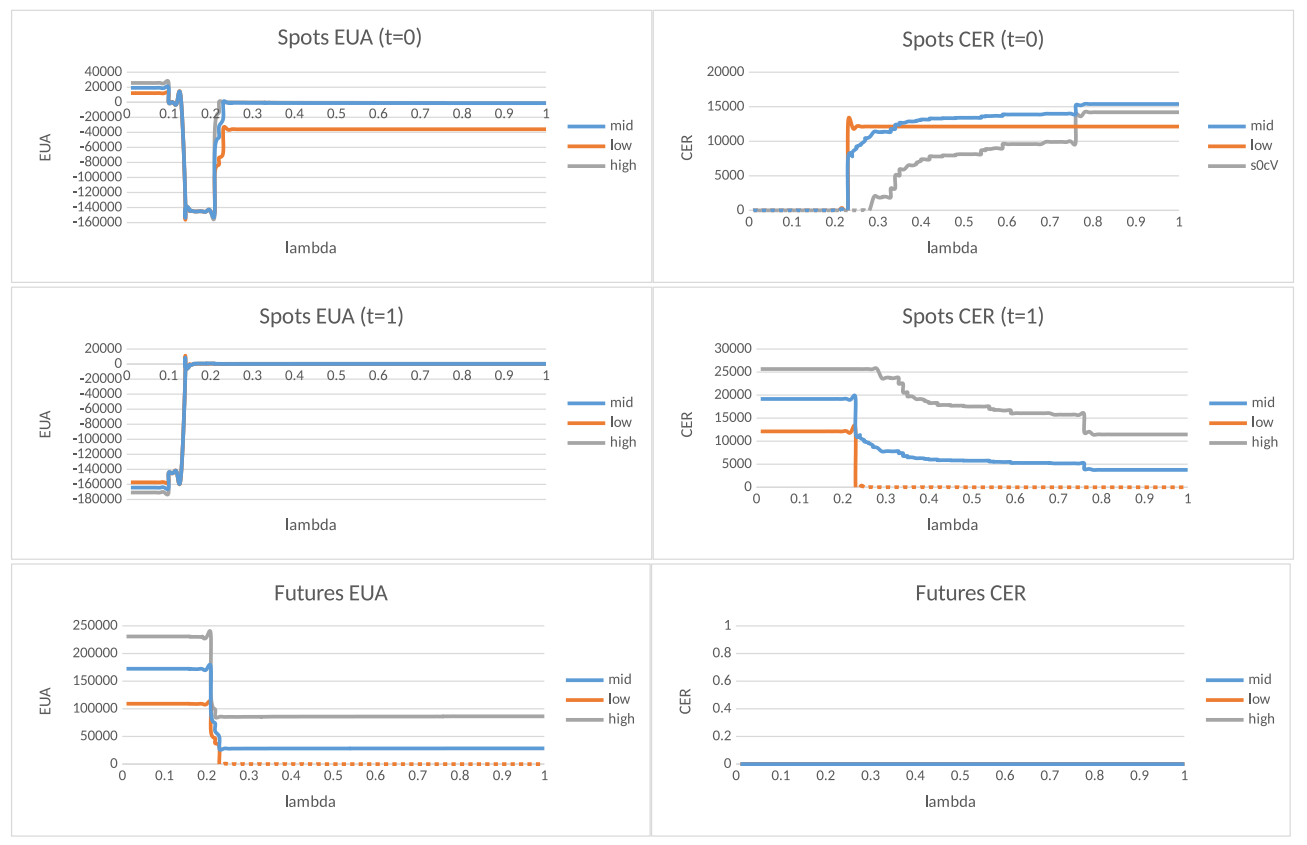

Fig. 3. Results by variable.

\section{CONCLUSIONS}

The goal of our paper was to discover which combination of carbon derivatives is optimal for a real-life risk averse Czech steel company, deciding according to the Mean-CVaR criterion. As we did not know the level of risk aversion of the company, we conducted the analysis for all possible weightings of mean and CVaR. Moreover, we took three scenarios of demand into account.

As expected, we found that the derivatives are useful for risk reduction, but, surprisingly, only futures, but no options, were recommended by the model under any combination of the parameters.

The question is, to what extent these results depend on the particular stochastic model we had chosen. Our model looks quite realistic, the volatilities of the spots roughly correspond to the past ones, for instance. However, the model predicts a positive expected return of the EUAs, which no way follows from the estimation but rather from our assumptions. As the estimation of the trend, hence of the price increase, is virtually impossible due to the high volatility of the spots, it would be useful to take the trend as another varying parameter of the analysis in addition to the risk aversion and the demand. This, however, would make the results too complex to be discussed in a single paper. Thus, dropping another varying parameter in favour of the trend and making a similar analysis might be worthwhile.

Another simplification we made was that we had considered only a single period of time; thus, we could neither model inter-period banking and/or borrowing of the 
Middle demand

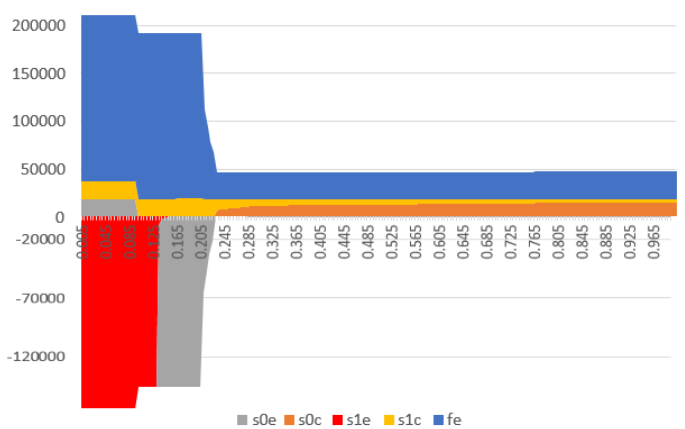

Middle demand

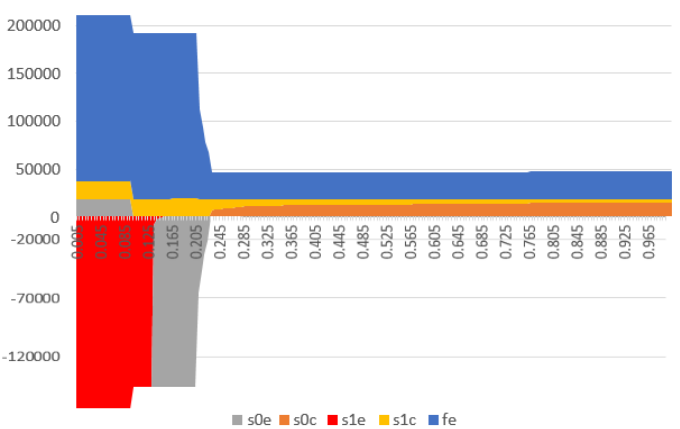

High demand

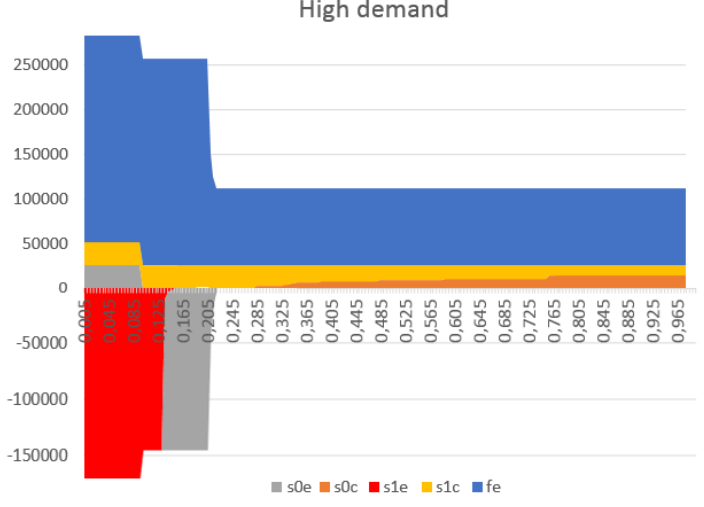

Fig. 4. Results by scenario. 
permits, nor study combinations of futures with various strike prices. Taking these and other dynamic aspects into account seems to be another promising direction for further research.

Even as it stands, however, our study may provide some insight regarding the optimal reduction of financial risks which stems from their emission obligations.

\section{A. APPENDIX - LINEARIZATION OF THE DECISION PROBLEM}

In the Appendix, we describe the linearization of problem (2). Even though the technique of linearization of Mean-CVaR problems into linear ones is well known and standard, we find useful to describe its application to 2 for the sake of replicability of our research.

Lemma 1. For any $\lambda \geq 0$,

$$
(1-\lambda) \mathbb{E}(Z)-\lambda \mathrm{CVaR}(-\mathrm{Z})=\max _{\mathrm{u}}\left[\mathbb{E} g_{\kappa, 1-\lambda}(Z+u)-u\right]
$$

where

$$
\kappa=1-\lambda+\frac{\lambda}{\alpha}
$$

Proof. We have, using [10, Theorem 10, that

$$
\begin{gathered}
(1-\lambda) \mathbb{E}(Z)-\lambda \operatorname{CVaR}_{\alpha}(-Z) \\
=(1-\lambda) \mathbb{E} Z-\lambda \min _{u}\left(u+\frac{1}{\alpha} \mathbb{E}[-Z-u]^{+}\right) \\
=(1-\lambda) \mathbb{E} Z+\lambda \max _{u}\left(-u-\frac{1}{\alpha} \mathbb{E}[-Z-u]^{+}\right) \\
=\max _{u}\left[(1-\lambda) \mathbb{E}(Z)-\lambda u-\frac{\lambda}{\alpha} \mathbb{E}[-Z-u]^{+}\right] \\
=\max _{u}\left[(1-\lambda) \mathbb{E}(Z)+(1-\lambda) u-u-\frac{\lambda}{\alpha} \mathbb{E}[-Z-u]^{+}\right] \\
=\max _{u}\left[(\lambda-1) \mathbb{E}[-Z-u]-u-\frac{\lambda}{\alpha} \mathbb{E}[-Z-u]^{+}\right] \\
=\max _{u}\left[\mathbb{E} g_{\lambda-1,-\kappa}(-Z-u)-u\right] \\
=\max _{u}\left[\mathbb{E} g_{\kappa, 1-\lambda}(Z+u)-u\right] .
\end{gathered}
$$

Using the Lemma, we may reformulate (2) as

$$
\begin{aligned}
\max _{y, \xi, u} & {\left[\mathbb{E}\left(g_{\kappa, 1-\lambda}(V(y, \xi)+u)-u\right]\right.} \\
& \text { s.t. }(P),(L),(F),(G),(E),(C),(S)
\end{aligned}
$$

Proposition 2. Problem 5 is convex. 
Proof. As both $B$ and $g_{1+\rho, 1+\iota}\left(E_{0}\right)$ are piecewise linear concave (p.l.c.) and $E_{1}$ and $F$ are linear, the argument of $g_{1+\sigma, 1}$ in 11 is p.l.c. Thus and because $g_{1+\sigma, 1}$ is p.l.c., $V+u$ is p.l.c. Therefore and because $g_{\kappa, 1-\lambda}$ is p.l.c., $g_{\kappa, 1-\lambda}(V+u)$ is p.l.c. so $\mathbb{E} g(V+u)$ is concave. As the feasibility set is clearly polyhedral, the problem is convex.

Lemma 3. Let $x$ and $y$ be real variables of a maximization decision problem such that the objective function is strictly increasing in $x$. Let the only constraint including variable $x$ be of the form

$$
x=\min (\alpha y, \gamma+\beta y)
$$

for some constants $\alpha, \beta, \gamma$. Then (6) is equivalent to

$$
x \leq \alpha y, \quad x \leq \gamma+\beta y .
$$

Proof. The validity of the assertion is obvious.

Proposition 4. If the distribution of $\left(M^{E}, M^{C}, P_{1}^{E}, P_{1}^{C}\right)$ is discrete, defined by

$$
\begin{gathered}
\mathbb{P}\left[M^{E}=m_{i}^{E}, M^{C}=m_{i}^{C}, P_{1}^{E}=\pi_{i}^{E}, P_{1}^{C}=\pi_{i}^{C}\right]=q_{i}, \quad q_{i}>0, \quad 1 \leq i \leq s, \\
\sum_{i=1}^{s} q_{i}=1,
\end{gathered}
$$

then (5) is equivalent to

$$
\begin{aligned}
& \max _{y, \xi, u, x, v, e, b} \quad\left[\sum_{i=1}^{s} q_{i} x_{i}-u\right] \\
& y, b \in \mathbb{R}^{n}, \xi \in \mathbb{R}^{6+k+l}, u, e \in \mathbb{R}, x, v \in \mathbb{R}^{s} \\
& \text { s.t. }(P),(L),(F),(G),(E),(C),(S) \\
& \quad x \leq(1-\lambda)(v+u), \quad x \leq \kappa(v+u) \\
& \quad v \leq z, \quad v \leq(1+\sigma) z \\
& z=e-f^{E} m^{E}-f^{C} m^{C}+D^{T} \xi+p^{T} b-(1+\iota) c^{T} y \\
& e \leq(1+\rho) \gamma^{T} \xi, \quad e \leq(1+\iota) \gamma^{T} \xi, \\
& b \leq d, \quad b \leq y
\end{aligned}
$$

where $m=\left(m_{i}\right)_{i \leq s}, D=\left(\delta\left(\pi_{i}^{E}, \pi_{i}^{C}\right)\right)_{i \leq s}$,

$$
\gamma=\left[\begin{array}{c}
-p_{0}^{E} \\
-p_{0}^{C} \\
0 \\
0 \\
0 \\
0 \\
-p_{0}^{c, K_{1}} \\
\cdots \\
-p_{0}^{c, K_{k}} \\
-p_{0}^{p, L_{1}} \\
\cdots \\
-p_{0}^{p, L_{l}}
\end{array}\right], \quad \delta\left(\pi^{E}, \pi^{C}\right)=\left[\begin{array}{c}
0 \\
0 \\
-\pi^{E} \\
-\pi^{C} \\
-p_{0}^{E, f} \\
-p_{0}^{C, f} \\
-\min \left(\pi^{E}, K_{1}\right) \\
\cdots \\
-\min \left(\pi^{E}, K_{k}\right) \\
\max \left(\pi^{E}, L_{1}\right) \\
\cdots \\
\max \left(\pi^{E}, L_{l}\right)
\end{array}\right] .
$$


Proof. If we denote $S$ the feasibility set of (5), we may write

$$
\begin{aligned}
\max _{(y, \xi, u) \in S}\left[\mathbb{E} g_{\kappa, 1-\lambda}(V(y, \xi)-u)-u\right] & \\
=\max _{(y, \xi, u) \in S}\left[\sum_{i=1}^{s} q_{i} g_{\kappa, 1-\lambda}(V(y, \xi ;\right. & \left.\left.\left.m_{i}^{E}, m_{i}^{C}, \pi_{i}^{E}, \pi_{i}^{C}\right)-u\right)-u\right] \\
& =\max _{(y, \xi, u) \in S, x, v \in \mathbb{R}^{s}, e \in \mathbb{R}, b \in \mathbb{R}^{n}}\left[\sum_{i=1}^{s} q_{i} x_{i}-u\right]
\end{aligned}
$$

subject to

$$
\begin{gathered}
x_{i}=g_{\kappa, 1}\left(v_{i}-u\right), \quad 1 \leq i \leq s, \\
v_{i}=g_{1+\sigma, 1}\left(z_{i}\right), \quad z_{i}=e-m_{i}^{E} f^{E}-m_{i}^{C} f^{C}+\delta_{i}^{T} \xi+p^{T} b-(1+\iota) c^{T} y, \quad 1 \leq i \leq s, \\
e=g_{1+\rho, 1+\iota}\left(\gamma^{T} \xi\right), \quad b=\min (d, y) .
\end{gathered}
$$

Thus, the Proposition follows from Lemma 3 used gradually to transform these constraints into $(\mathrm{B}),(\mathrm{M}),(\mathrm{Z}),(\mathrm{V}),(\mathrm{X})$.

\section{ACKNOWLEDGEMENT}

This work was supported by grant No. GA 16-01298S of the Czech Science Foundation. The support is kindly acknowledged.

(Received February 8, 2017)

\section{REFERENCES}

[1] P. Artzner, F. Delbaen, J.-M. Eber, and D. Heath: Coherent measures of risk. Math. Finance 9 (1999), 3, 203-228. DOI:10.1111/1467-9965.00068

[2] E. A. Benz and J. Hengelbrock: Liquidity and price-discovery in the european co2 futures market: An intraday analysis (working paper). University of Bonn, 2009.

[3] J. Chevallier: Econometric analysis of carbon markets: the European Union emissions trading scheme and the clean development mechanism. Springer Science and Business Media, 2011. DOI:10.1007/978-94-007-2412-9

[4] European Comission: Use of international credits. https://ec.europa.eu/clima/ policies/ets/credits_en

[5] EU Commission et al.: Directive 2003/87/ec of the european parliament and of the council of 13 october 2003 establishing a scheme for greenhouse gas emission allowance trading within the community and amending council directive 96/61/ec. Union, European 46 (2003), 32-46. DOI:10.1017/cbo9780511610851.034

[6] G. Daskalakis, D. Psychoyios, and R. N. Markellos: Modeling co 2 emission allowance prices and derivatives: Evidence from the european trading scheme. J. Banking Finance 33 (2009), 7, 1230-1241. DOI:10.1016/j.jbankfin.2009.01.001 
[7] X. Gong and S. X. Zhou: Optimal production planning with emissions trading. Oper. Res. 61 (2013), 4, 908-924. DOI:10.1287/opre.2013.1189

[8] J. C. Hull and S. Basu: Options, Futures, and Other Derivatives. Prentice Hall, New Jersey 2016. DOI:10.23874/amber/2016/v7/i1/121351

[9] P. Mazza and M. Petitjean: How integrated is the european carbon derivatives market? Finance Res. Lett. 15 (2015), 18-30. DOI:10.1016/j.frl.2015.07.005

[10] R. T. Rockafellar and S. Uryasev: Conditional value-at-risk for general loss distributions. J. Banking Finance 26 (2002), 7, 1443-1471. DOI:10.1016/s0378-4266(02)00271-6

[11] Bao-jun Tang, Cheng Shen, and Chao Gao: The efficiency analysis of the european co 2 futures market. Applied Energy 112 (2013), 1544-1547. DOI:10.1016/j.apenergy.2013.02.017

[12] M. Uhrig-Homburg and M. Wagner: Futures price dynamics of co2 emission allowances: An empirical analysis of the trial period. J. Derivatives 17 (2009), 2, 73-88. DOI:10.3905/jod.2009.17.2.073

[13] F. Zapletal and M. Šmíd: Mean-risk optimal decision of a steel company under emission control. Central Europ. J. Oper. Res. 24 (2016), 2, 435-454. DOI:10.1007/s10100-0150430-7

Martin Šmíd, Institute of Information Theory and Automation, The Czech Academy of Sciences, Pod Vodárenskou věží4, 18208 Praha 8. Czech Republic.

e-mail:smid@utia.cas.cz

František Zapletal, VSB - Technical University of Ostrava, Faculty of Economics, Sokolská 33, 702 00 Ostrava. Czech Republic.

e-mail: frantisek.zapletal@vsb.cz

Jana Hančlová, VSB - Technical University of Ostrava, Faculty of Economics, Sokolská 33, 702 00 Ostrava. Czech Republic.

e-mail: jana.hanclova@vsb.cz 